\title{
Development of Mineral Hybrid Fiber from Calcium Carbonate and Pulp Using Fluid-jet Cavitation
}

\author{
Moe Fukuoka*2, Toru Nakatani and Shisei Goto
}

NPi Research Laboratory, Nippon Paper Industries Co., Ltd. ${ }^{* 1}$

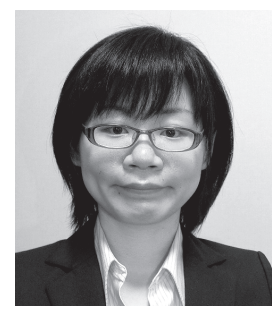

Moe Fukuoka

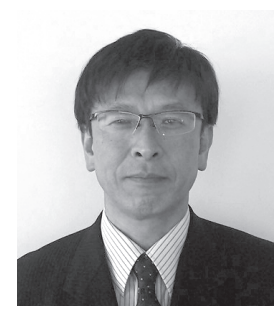

Toru Nakatani

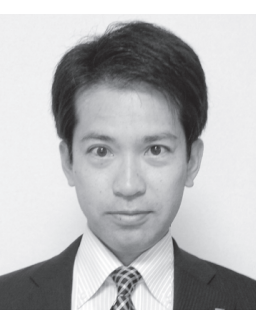

Shisei Goto

\begin{abstract}
Studies on the synthesis and characterization of a hybrid fiber (HF) consisting of precipitated calcium carbonate (PCC) and cellulose fiber were conducted. The PCC-HF has been prepared by modifying the carbon dioxide $\left(\mathrm{CO}_{2}\right)$ method for PCC synthesis under high-pressure fluid-jet cavitation in hardwood bleached kraft pulp (HBKP) slurry. Scanning electron microscopy (SEM) observations of the HF showed that almost all fiber surfaces were covered with nano-size PCC particles. By using this PCC-HF as raw materials, the handsheet containing more than 50\% PCC by weight with a high first-pass ash retention (greater than $80 \%)$. Unlike ordinary paper such as printing paper, the sheet showed remarkable physical properties, such as high flexibility without crumbling despite extreme high ash content. The SEM images from a cross section of the sheet containing $74 \%$ PCC revealed that a very little number of fibers embedded in PCC layer. This implied that the bonding mechanism of HF sheet was different from ordinary paper. In order to clarify the mechanism, the slurry of PCC-HF for handsheet making was separated into two fractions, long fibers and fines, by using the Dynamic Drainage Analyzer (DDA). It was found that the nano-size PCC was attached onto not only the surfaces of the long fibers but also those of fiber fines. Thus, the PCC-hybrid fiber fines contributed sheet properties and high ash retention.
\end{abstract}

Keywords : cavitation, composite, high filler, calcium carbonate, filler retention

\section{Introduction}

One of the methods to improve functional properties of paper is adding inorganic fillers to the paper stock. For instance, brightness and opacity of printing paper are improved through filling of calcium carbonate particles. Although these inorganic fillers generally enhance func-

\footnotetext{
${ }^{* 1}$ 5-21-1 Oji, Kita-ku, Tokyo 114-0002, Japan

${ }^{* 2} \mathrm{E}$-mail : fuchise-moe@nipponpapergroup.com
}

tional properties, the use of a high percentage of fillers will also result in some undesirable effects principally a decrease in first pass retention and an increase in usage of chemicals. Homogeneous distribution of the filler in paper matrix is required to give better functional paper properties. However, the control of the distribution of them is difficult to achieve just by simple addition of them because they locally produce aggregates in the paper matrix. To solve this problem, filler particles especially calcium carbonate can be loaded into fiber lumen 
by the methods referred to as "lumen-loading" or "fiberloading". Kumar et al. has published a review on the historical development of those composites ${ }^{11}$. In particular, precipitated calcium carbonate (PCC) has been studied as the inorganic substance for the preparation of composites. For example, Craig et al. synthesized a composite of PCC and pulp by the "liquid.liquid method" to add sodium carbonate to the pulp pretreated with calcium chloride $^{2)}$. Srivatsa et $a l^{3}$. and Yoshida et $a l .^{4)}$ independently reported that they developed a method of depositing PCC on the fiber surface by the "gas-liquid method" to infuse carbon dioxide gas to the pulp after mixing calcium hydroxide with the pulp. However, in any studies, the particle size of PCC was not specifically mentioned. We speculate that they can be synthesized only in micron-order particles and are interested in the characteristics of composites of nano-size PCC and pulp.

In recent years, research that raised the term "nanotechnology" is active because, the physical and chemical properties remarkably change when particle size is smaller than $100 \mathrm{~nm}$. Even if a material is of a generalpurpose item, unexpected properties can appear when the particle dimensions are greatly diminished, and therefore a movement to find a new method of utilizing materials with unique characteristics prevails. If such nanoparticles are internally filled in a paper matrix, they are more likely to pass through the pores between pulp fibers in comparison with micron-size particles. Also, nanoparticles having large specific surface area consume much more chemicals. As a result, they can deteriorate first pass retention. Furthermore, because nanomaterials are very adhesive and have strong tendency to agglomerate, control of dispersion and agglomeration is essential when using nanoparticles ${ }^{5}$. From such a viewpoint, we developed a hybrid fiber (HF) consisted of PCC nanoparticles and pulp. It was found that PCC nanoparticles are evenly deposited on the surface and in the lumen of fibers. PCC-HF has desirable effects not only to increase first pass retention but also to handle nanoparticles in a distributed in the paper matrix.

Several methods are reported to synthesize PCC in nano-order (hereinafter called as nano PCC). For example, mechanically grinding method, finely dispersing of bubbles in the "gas-liquid" method, and controling of particle diameter by chemical addition. Kojima synthesized PCC particles with a primary diameter of $<40 \mathrm{~nm}$ by concurrently irradiating ultrasonic wave and injecting carbon dioxide gas to calcium hydroxide suspension ${ }^{6}$. Sonawane et al. prepared PCC in the order of dozens of nanometers by cavitation ${ }^{7}$. Cavitation is a phenomenon in which rapid changes of pressure in a liquid lead to the formation of small vapor-filled cavities, in places where

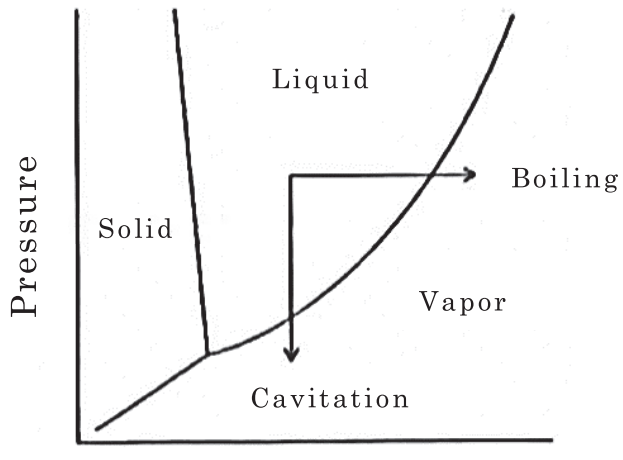

Temperature

Fig. 1 Vapor pressure curve ${ }^{9)}$

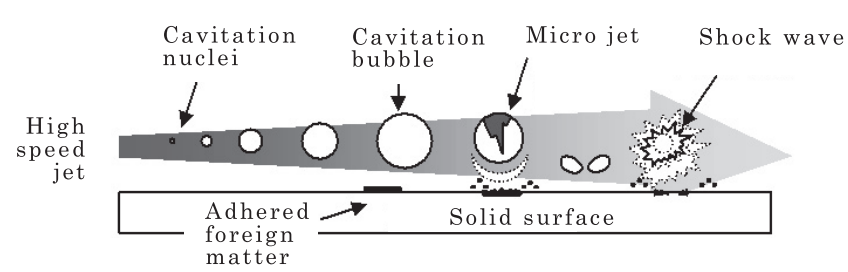

Fig. 2 Illustrate of Cavitation bubbles ${ }^{10)}$

the pressure is relatively low. When subjected to higher pressure, these cavities, called "bubbles" or "voids" of 0.1-0.2 mm diameter (Fig. 1), collapse and can generate an intense shock wave in the order of several GPa pressure (Fig. 2). Artificial cavitation for industrial application is formed by various methods such as, (1) fluid-jet, (2) ultrasonic wave oscillator, (3) combination of ultrasonic oscillator and horn-shaped amplifier, (4) mechanical agitation, (5) laser beam irradiation (Table 1). Among them, we selected (1) fluid-jet cavitation and studied application for deinking of recycled pulps because it is easy to scale up $^{8}$.

In this paper, we report a hybrid fiber (HF) consisted with pulp and precipitated calcium carbonate (PCC) nanoparticles obtained by synthesizing PCC by treating the pulp with fluid-jet cavitation. The resultant HF was characterized by first pass retention experiments and handsheet tests to compare with ordinary PCC filled paper.

\section{Experimental}

\subsection{Raw Materials}

- Bleached hardwood kraft pulp ; HBKP (unrefined, $\mathrm{CSF}=495 \mathrm{~mL}$, from mill of Nippon Paper Industries)

We parchased following commercial products.

- Calcium hydroxide (industrial special grade)

- Carbon dioxide gas (liquified carbon dioxide)

- Cationic retention aid (molecular weight : 15,000,000, charge density : $+2.1 \mathrm{meq} / \mathrm{g})$

- Anionic retention aid (molecular weight : 14,000,000, charge density : $-1.8 \mathrm{meq} / \mathrm{g}$ )

- PCC (primary diameter $1.2 \mu \mathrm{m}$ ) 
Table 1 Characteristics of method to generate cavitation

\begin{tabular}{|c|c|c|c|}
\hline & Principle of generation & Vibration frequency & Caracteristics \\
\hline Liquid jet & $\begin{array}{l}\text { decrease in local pressure } \\
\text { by jet }\end{array}$ & $\begin{array}{l}\text { broad frequency } \\
\text { (variable) }\end{array}$ & $\begin{array}{l}\text { high intensity, } \\
\text { easy to controll, } \\
\text { easy to scale-up }\end{array}$ \\
\hline Ultrasonic vibrater & $\begin{array}{l}\text { fluctuation of sound } \\
\text { pressure }\end{array}$ & intrinsic frequency & $\begin{array}{l}\text { low intensity, high } \\
\text { energy consumption }\end{array}$ \\
\hline $\begin{array}{l}\text { Ultrasonic transducer } \\
\text { with horn amplifier }\end{array}$ & $\begin{array}{l}\text { fluctuation of sound } \\
\text { pressure }\end{array}$ & intrinsic frequency & $\begin{array}{l}\text { high intensity, difficult to } \\
\text { scale-up }\end{array}$ \\
\hline mechanical mixing & $\begin{array}{l}\text { decrease in local pressure } \\
\text { by shear }\end{array}$ & broad frequency & difficult to controll \\
\hline laser irradiation & phase interference & intrinsic frequency & high cost \\
\hline
\end{tabular}

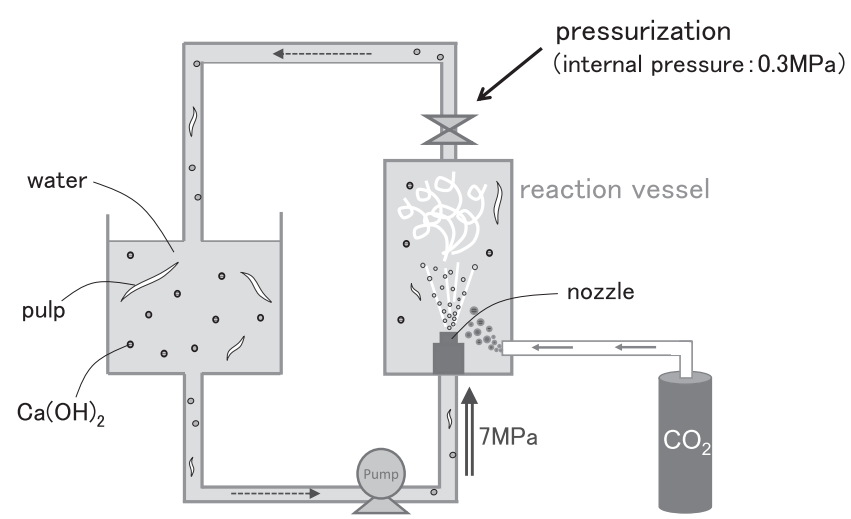

Fig. 3 A schematic diagram of the reaction apparatus used in this study

\subsection{Instrument}

- Cavitating Jet Device (CV-jet device $)^{8)}($ Fig. 3) : nozzle diameter $1.5 \mathrm{~mm}$

- Dynamic Drainage Analyzer (DDA, Ab Akribi Kemikonsulter) : 60 mesh

- Scanning Electron Microscope (SEM, JSM-6700, JEOL)

- Surface Area Analyzer (GEMINI2360, Shimadzu)

\subsection{Testing Protocol}

\subsubsection{Synthesis of hybrid fibers}

Calcium hydroxide $(89 \mathrm{~g})$ and HBKP $(120 \mathrm{~g})$ was dispersed in tap water $(12 \mathrm{~L})$ at room temperature and was put into a reactor of $\mathrm{CV}$-jet device. A pump was run for 3 minutes to fill the reactor with the pulp slurry without operating $\mathrm{CV}$-jet device. Initial $\mathrm{pH}$ was 12.8 . The $\mathrm{CV}$-jet device was ran with the conditions of upstream pressure at $7 \mathrm{MPa}$ and downstream pressure at $0.3 \mathrm{MPa}$ to generate fluid-jet cavitation. carbon dioxide gas was introduced into the reactor $(5 \mathrm{~L} / \mathrm{min})$ for about $6 \mathrm{~min}$ utes. The reaction was terminated when $\mathrm{pH}$ reached 7.4 and reaction mixture was cooled down to $20^{\circ} \mathrm{C}$ by putting plastic bags containing crushed ice to get PCC-HF. The PCC-HF slurry was filtered with Advantec No.5B filter paper. Ash content of PCC-HF was calculated from the ash content of filtrate based on JIS P $8251: 2003$. Theoretical value was $50 \%$.

\subsubsection{Synthesis of nano PCC}

Without using HBKP, experimental protocol of PCC synthesis was the same as described in the section 2.3.1

\subsubsection{SEM observation of PCC-HF sheet}

A sample $(10 \mathrm{~mL})$ of PCC-HF slurry prepared in the section 2.3.1 was filtered with filter paper (Advantec No.2) in vacuum and washed with ethanol to prepare a specimen for SEM observation. A copper tape was pasted on a brass sample table, on which the sample was dropped, air dried and deposited with osmium vapor. For the observation of the sheet surface, the sheet was cut in $3 \mathrm{~mm} \times 3 \mathrm{~mm}$ and placed on the brass table facing up a wire side.

\subsubsection{Preparation of handsheets}

\section{1) $\mathrm{PCC}-\mathrm{HF}$ sheet}

PCC-HF slurry obtained in the section 2.3.1 was adjusted at $0.5 \%$ consistency (Fig. 4). The slurry (250 $\mathrm{mL})$ was put into a plastic cup $(300 \mathrm{~mL})$, and agitated at $600 \mathrm{rpm}$. cationic retention aid (100 ppm/solid) and anionic retention aid $(100 \mathrm{ppm} / \mathrm{solid})$ were added into the slurry. Handsheets of $60 \mathrm{~g} / \mathrm{m}^{2}$ were prepared using 150 mesh (JIS P 8222).

\section{2) Ordinary filled sheet}

PCC was added to $0.5 \%$ HBKP slurry to make $50 \%$ PCC consistency. This mixture is hereinafter referred to as mixture of fiber and PCC or abbreviated as PCC-MF. Two kinds of calcium carbonate fillers were used to prepare handsheets of PCC-MF $\left(60 \mathrm{~g} / \mathrm{m}^{2}\right)$ independently : 


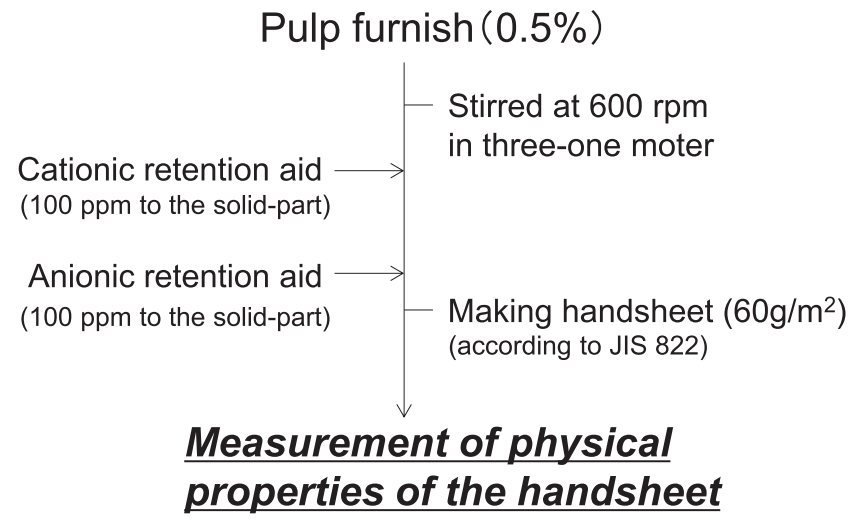

Fig. 4 Schematics of experiment of making handsheets

one was PCC nanoparticles hereinafter referred to as nano PCC, synthesized by the method described in the section 2.3.2, and the other was commercially available PCC referred to as micro PCC.

3) Bleached Hardwood Kraft Pulp Sheet

Handsheets of HBKP were prepared in the same method as described in the section

\subsubsection{Fractionation of fibers and fines}

The slurry samples obtained in the section 2.3 .4 were adjusted at $0.1 \%$ consistency by tap water with no retention aids. Slurry $(400 \mathrm{~mL})$ was put into DDA, agitated at $800 \mathrm{rpm}$ for 20 seconds, and dewatered under the vacuum of 0.3 bar for 20 seconds. Ash contents of residues was measured with the same method described in the section 2.3.1. Residue (fiber fraction) and filtrate

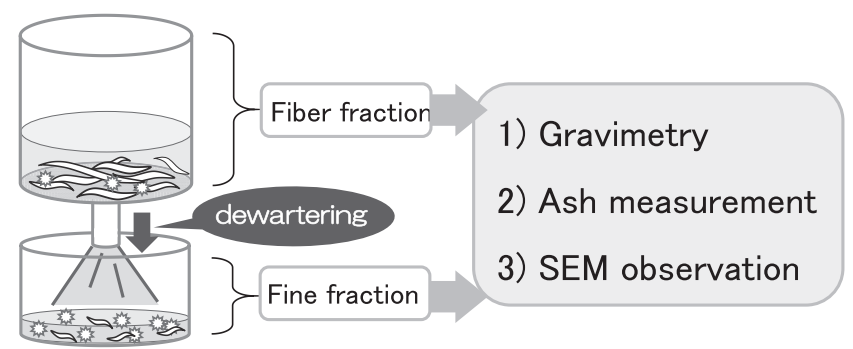

Fig. 5 Illustrate of fractionation with Dynamic Drainage Analyzer (fines fraction) were observed with SEM (Fig. 5). SEM observation was performed in the same method as desctived in the section 2.3.3.

\section{Results}

\subsection{SEM observation of PCC-HF handsheets}

The ash content of PCC-HF handsheet was $48 \%$. The SEM observation of the surface of the sheet revealed that inorganic particles (averaged primary diameter was about $80 \mathrm{~nm}$ ) densely covered the surface of pulp (Fig. $6)$.

\subsection{Properties of the Handsheet}

Physical properties of the handsheets prepared from the PCC-HF and PCC-MF slurries are shown in Table 2. Among them, several characteristic properties such as scattering coefficient, BET surface area and breaking length are summarized in in Fig. $7 \sim 9$. The scattering coefficient of PCC-HF sheets was about $50 \%$ of the micro PCC-MF sheets and was almost the same level of HBKP sheets. The BET surface area of PCC-HF sheets was slightly larger than nano PCC-MF and was three times larger than micro PCC-MF. The breaking length of the PCC-HF sheets was 20-30 \% higher than nano $\mathrm{PCC}-\mathrm{MF}$ and micro PCC-MF. Other properties such as tear strength and stiffness of the PCC-HF sheets were higher than that of PCC-MF sheets. Unlike ordinary paper, PCC-HF sheet had remarkable physical properties, such as high flexibility without crumbling and less dusting tendency despite extreme high ash content.

\subsection{SEM observation of sheet surface}

The surface of handsheets prepared from PCC-HF and PCC-MF slurries was observed with SEM (Fig. 10). For the PCC-HF sheet, inorganic fillers observed as white part were found to be deposited on the fiber observed in gray. On the other hand, for the PCC-MF sheets, most of inorganic fillers were located between fibers as agglomerates and only a little amount of fillers was observed on fiber surfaces.
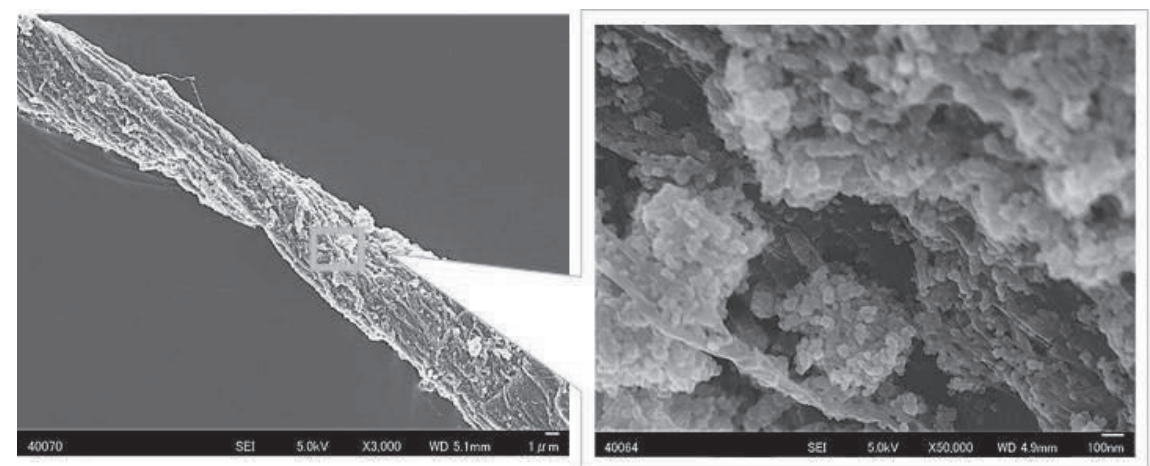

Fig. 6 SEM images of the PCC-HF obtained by hybridization of PCC and HBKP (left ; $\times 3,000$, right ; $\times 50,000)$ 
Table 2 Comparison of physical properties of the handsheets made from PCC-HF, mixture of nano PCC and HBKP, mixture of micro PCC and HBKP, and raw HBKP

\begin{tabular}{llcccc}
\hline $\begin{array}{l}\text { Raw material } \\
\text { (Ash content of raw material) }\end{array}$ & $\begin{array}{c}\text { PCC-HF } \\
(50 \%)\end{array}$ & $\begin{array}{c}\text { Nano PCC + HBKP } \\
(50 \%)\end{array}$ & $\begin{array}{c}\text { Micro PCC + HBKP } \\
(50 \%)\end{array}$ & $\begin{array}{c}\text { HBKP } \\
(0 \%)\end{array}$ \\
\hline Basis weight & $\mathrm{g} / \mathrm{m}^{2}$ & 62.4 & 57.6 & 61.5 & 64.4 \\
Thickness & $\mu \mathrm{m}$ & 106 & 98 & 110 & 122 \\
Density & $\mathrm{g} / \mathrm{cm}^{3}$ & 0.59 & 0.59 & 0.56 & 0.53 \\
Ash content & $\%$ & 44.6 & 44.8 & 48.6 & 0.4 \\
\hline Whiteness & $\%$ & 85.9 & 90.0 & 91.3 & 86.2 \\
Opacity & $\%$ & 81.8 & 78.5 & 88.3 & 78.4 \\
Specific scattering coefficient (S value) $)$ & $\mathrm{m}^{2} / \mathrm{kg}$ & 48.1 & 48.6 & 85.3 & 41.2 \\
Air resistance & $\mathrm{sec}$ & 10 & 5 & 1 & 2 \\
Smoothness & $\mathrm{sec}$ & 8 & 10 & 9 & 6 \\
\hline Specific tear strength & $\mathrm{mN} /\left(\mathrm{g} / \mathrm{m}^{2}\right)$ & 1.6 & 1.3 & 1.5 & 6.6 \\
L\&W stiffness & $\mu \mathrm{N} \cdot \mathrm{m}$ & 72 & 42 & 63 & 211 \\
Breaking length & $\mathrm{km}$ & 1.3 & 1.0 & 1.1 & 4.1 \\
Elongation & $\mathrm{mm}$ & 1.3 & 1.3 & 1.5 & 2.8 \\
\hline Specific surface area (BET) & $\mathrm{m}^{2} / \mathrm{g}$ & 15 & 14 & 5 & 2 \\
\hline
\end{tabular}

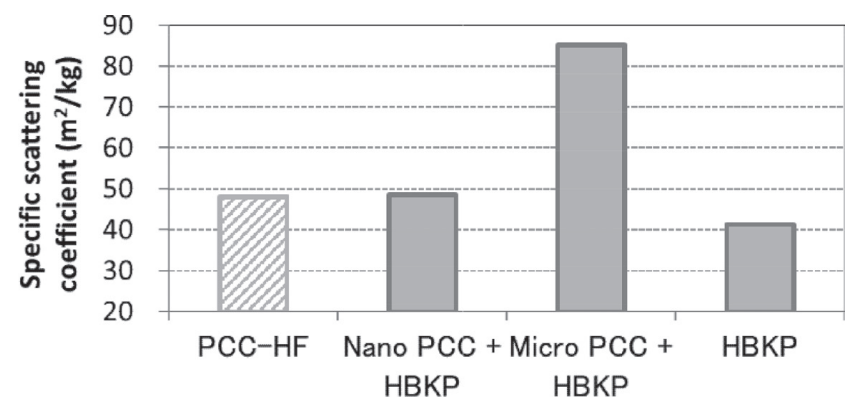

Fig. 7 Specific scattering coefficient of the handsheets

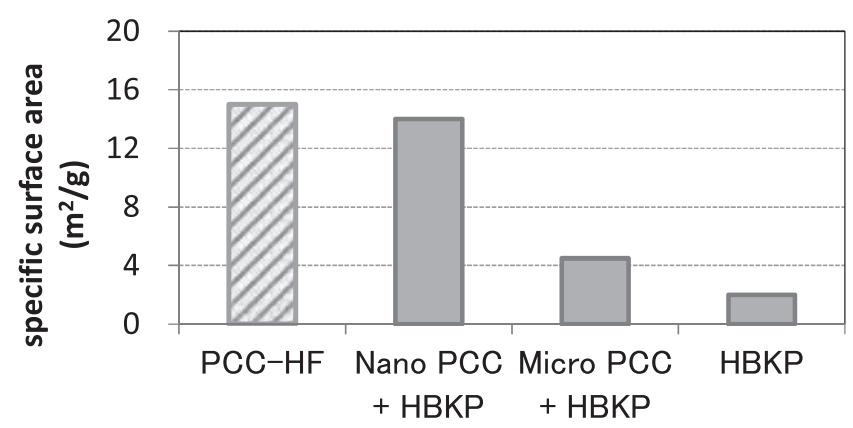

Fig. 8 Specific surface area of the handsheets

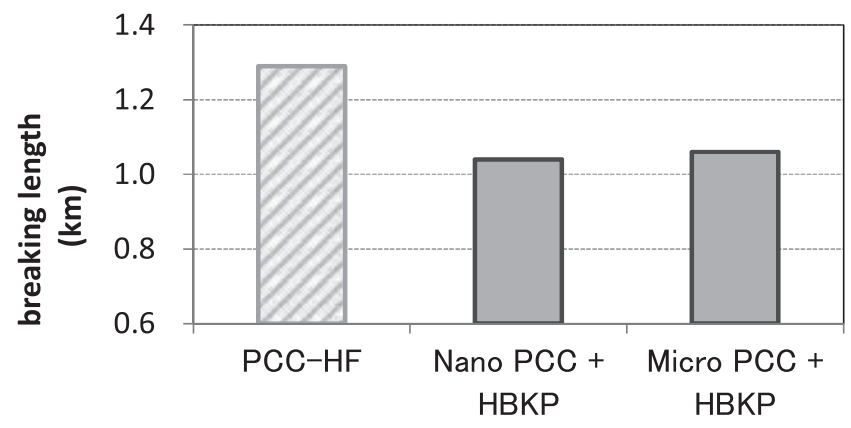

Fig. 9 Breaking length of the handsheets

\subsection{Fractionation of fibers and fiber fines by DDA}

The ash retention of the PCC-HF and PCC-MF were calculated from the ash content of DDA residues. It was found that the ash retention of the PCC-HF was 20-30 point higher than that of PCC-MF (Fig. 11). The SEM observation for the residues and filtrate obtained by DDA experiment revealed that a lot of PCC particles deposited on the surface of long fiber and fiber fines of PCC-HF. On the other hand, for the PCC-MF, a little amount of PCC was observed on the surface of long fiber and fiber fines.

\section{Discussion}

According to the physical properties of the sheets listed in Table 2, PCC-HF sheet had slightly lower scattering coefficient, slightly larger surface area and $30 \%$ higher physical strength than the PCC-MF sheet. From these results, we inferred that the particle size of the PCC led to the low scattering coefficient and the high surface area. On the other hand, the composite consisting of pulp and PCC led to the high strength of the PCC-HF sheet. Besides, the results of retention experiment descrived in the section 3.4 revealed that the ash retention of PCC-HF was much higher than that of PCC-MF.

The SEM observation descrived in the section 3.4 revealed that PCC nanoparticles were not only deposited on the surface of long fibers, but also agglomerated with fibrillated fiber fines as shown in Fig. 13. We proposed the mechanism of filtration to produce PCC-HF and PCC-MF as shown in Fig. 14. When a slurry of fibers, fines, and PCC is filtered to produce PCC-MF sheets, small particles are not trapped in a pulp mat and can easily pass through filter pores, to give filtrate containing large amount of fiber fines and fillers. In contrast, producing the PCC-HF sheet, PCC-supported long fibers, aggregates of fiber fines and PCC cannot pass through the filter and to remain in the sheet matrix. Further studies are needed to find out why the PCC-HF sheets 


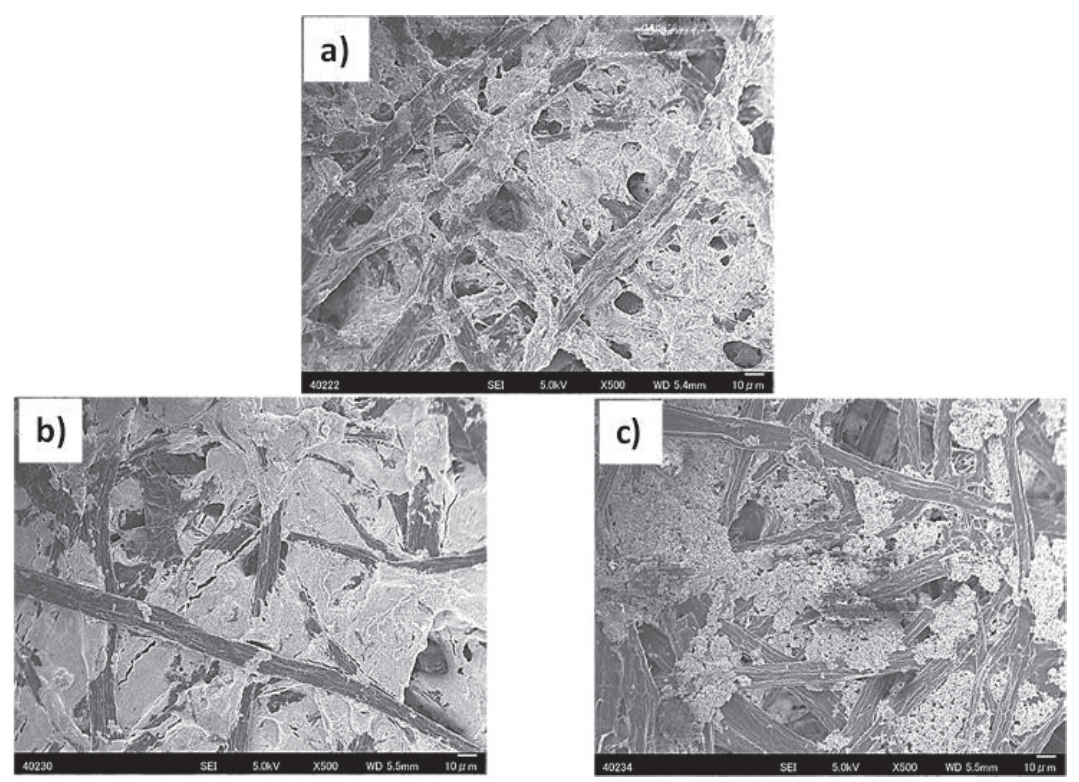

Fig. 10 SEM image of surface of handsheet from a) PCC-HF, b) mixture of nano PCC and HBKP, c) mixture of micro PCC and $\operatorname{HBKP}(\times 500)$

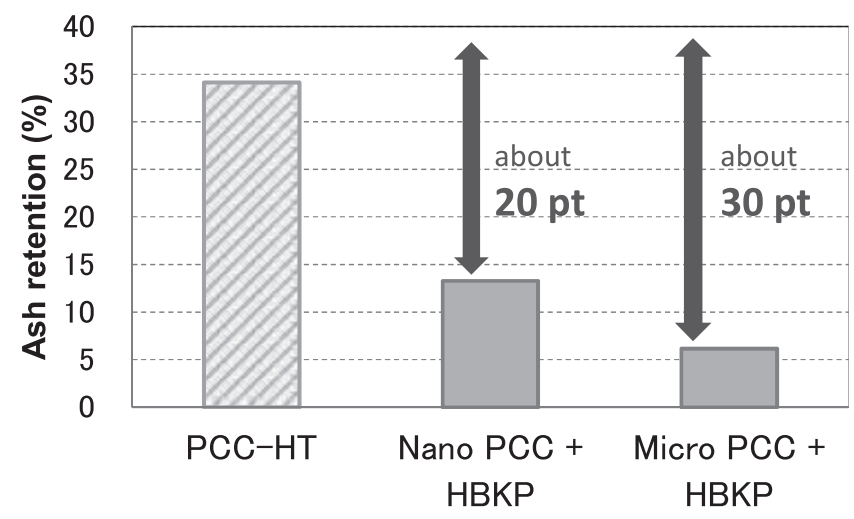

Fig. 11 Ash retention of the samples had higher physical properties than the PCC-MF sheets in detail. But at present, we would like to propose two hypothetical mechanisms. (1) The composite of pulp and PCC in the PCC-HF had just a little part where is mechanically extremely weak, because the SEM images of the PCC-HF showed that pulp and PCC homogeneously disperse. On the other hand, the PCC-MF showed that pulp and PCC separately existed in some parts. (2) The fiber fines in the PCC-HF contributed to the increase in the mechanical strength, since the aggregated fiber fines can easily remain in the sheet. Therefore, PCC-HF pa-

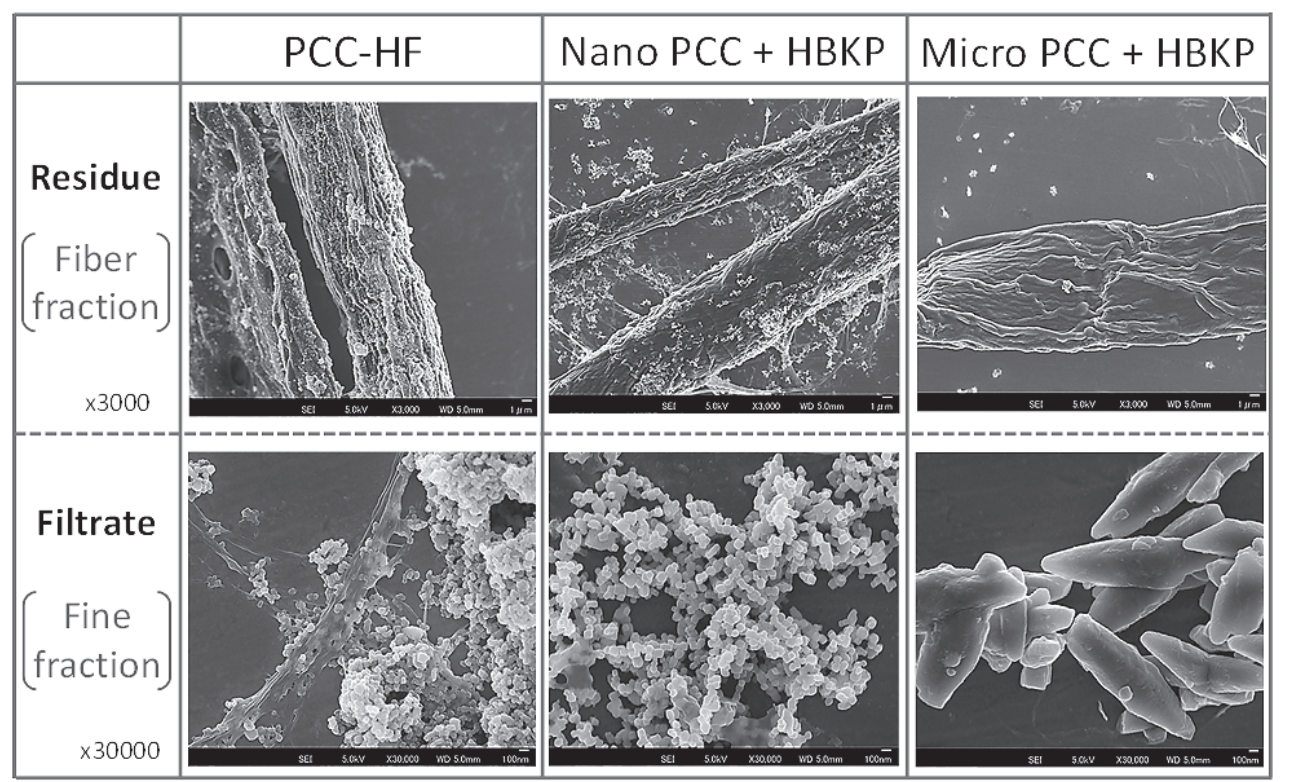

Fig. 12 SEM image of the samples after the fractionation by Dynamic Drainage Analizer 


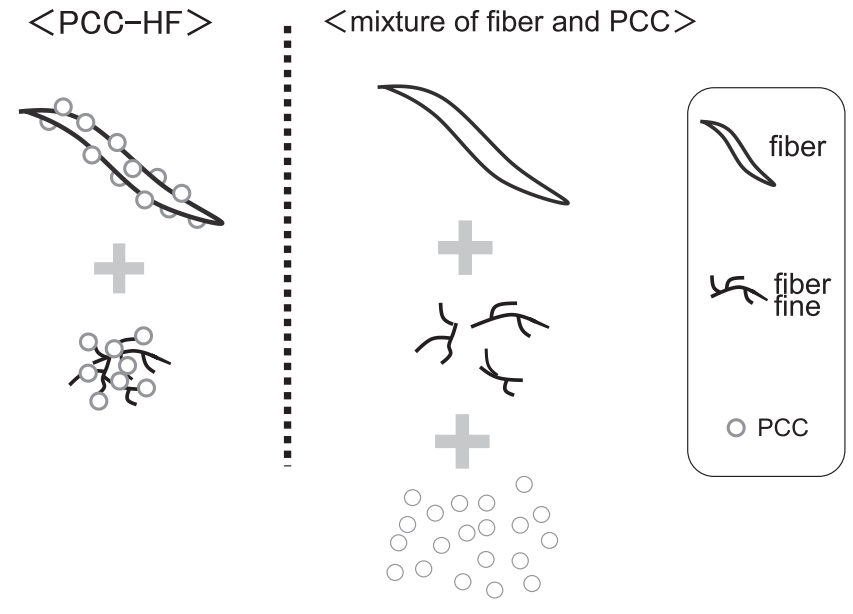

Fig. 13 Illustrate of PCC-HF and mixture of pulp and PCC
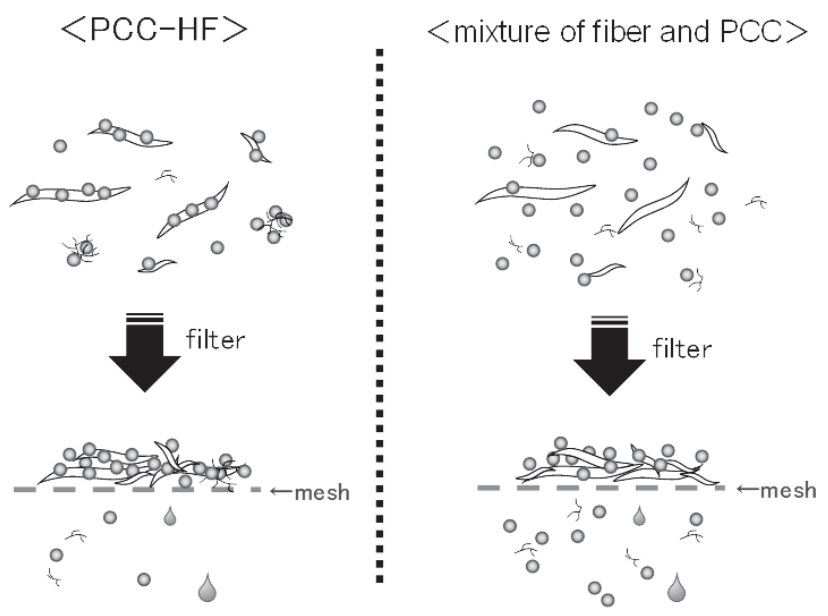

Fig. 14 Illustrate of PCC-HF and mixture of pulp and PCC

pers showed high mechanical strength even when the high ash content of around $50 \%$.

\section{Conclusions}

We discovered that the synthesis of PCC under highpressure fluid-jet cavitation in the presence of pulp pro- duces the hybrid fibers consisted of cellulose fiber of which surfaces were covered by nano-size PCC particles, i.e. PCC-HF. In addition, we found that the handsheets containing about $50 \%$ PCC by weight were easily prepared from the PCC-HF as raw materials. Unlike paper filled with PCC of ordinary-size (micro order), i.e. micro PCC-MF sheet, the obtained sheet showed characteristic physical properties, such as low scattering coefficient, large specific surface area, and high breaking length. In particular, the observed high breaking length is the most remarkable characteristics of the $\mathrm{PCC}-\mathrm{HF}$ sheets; the sheet made from conventional method with nano-size PCC (nano PCC-MF) showed low breaking length similar to the micro PCC-MF sheet. The slurry of PCC-HF was separated into two fractions, long fibers and fines, by using the Dynamic Drainage Analyzer (DDA) to determine the ash retention of $\mathrm{PCC}-\mathrm{HF}$ and $\mathrm{PCC}-\mathrm{MF}$. The ash retention of $\mathrm{PCC}-\mathrm{HF}$ was found to be 20-30 point higher than micro PCC-MF and nano PCC-MF. The SEM images of each fraction obtained from the PCC-HF revealed that nano-size PCC attached onto not only the surfaces of the long fibers but also those of fiber fines.

\section{References}

1) Pradeep Kumar, et al. : Bio Resources 6 (3), 35263546 (2011)

2) William L. : Craig, US2583548(1952)

3) Narendra R. Srivatsa, et al. : USOO5665205A

4) Yoshida T., et al. : JPS62162098A (1987)

5) Kamiya H., et al. : The micromeritics $55,12-18(2012)$

6) Kojima Y. : JP5467276B2

7) Shirish H. Sonawane, et al. : International Journal of Chemical Engineering (2010)

8) Shisei Goto, et al. : TAPPI Journal 13(9), 9-26(2014)

9) Kato H. : "Cavitation : Kiso to Saikin no Shinpo", Maki Shoten, Tokyo, Japan(1999)

10) Goto S. : Pulp \& Paper Symposium, 44th, The Society of Fiber Science and Technology, Tokyo, Japan (2009) 